\section{PPAD is not targeted as a citrullinated protein in rheumatoid arthritis, but remains a candidate for inducing autoimmunity}

We thank Dr Quirke and colleagues for their interest in our study. We have some concerns, however, that the authors' attempt to reconcile our work on Porphyromonas gingivalis peptidylarginine deiminase (PPAD) autocitrullination with their model of periodontal disease in rheumatoid arthritis (RA) misrepresents our data. ${ }^{12}$

The discovery that citrullination is the primary antigenic determinant recognised by autoantibodies in RA has generated considerable interest in the family of enzymes, peptidylarginine deiminases (PADs), that catalyse this post-translational protein modification. ${ }^{3}$ The identification of a bacterial PAD from $P$ gingivalis led to the initial hypothesis by Rosenstein $e t a l^{4}$ that infection with this periodontal pathogen may be aetiologically linked to RA.

Two distinct mechanisms have since been hypothesised by which PPAD may trigger loss of tolerance to citrullinated proteins and autoimmunity in RA-first, by acting as a citrullinating enzyme on bacterial and host proteins, ${ }^{45}$ and second, through the process of enzyme autocitrullination. ${ }^{6}$ In the absence of experimental evidence to support PPAD autocitrullination in $P$ gingivalis or in vivo, Quirke $e t a l^{2}$ posited a third mechanism in which PPAD acts as a substrate for human PADs, thereby becoming the target of a citrulline-specific antibody response. The first two hypotheses support $P$ gingivalis as a unique periodontal bacterium relevant to RA pathogenesis. The uniqueness of $P$ gingivalis for RA is lost, however, when considering the third hypothesis of bacterial proteins as putative substrates for human PADs. Under this premise (not supported by experimental data), human PADs may generate citrullinated antigens from any conceivable pathogen, making $P$ gingivalis expendable as a model organism. To strengthen the credibility of PPAD for inducing autoimmunity in RA, Quirke et al variably use these three hypotheses. ${ }^{2}$

Importantly, our study only addressed the question of PPAD autocitrullination as a possible link of periodontal disease and RA. ${ }^{1}$ This mechanism, in which tolerance to peptidyl-citrulline is presumably lost due to an immune response to autocitrullinated bacterial PPAD, was supported by two findings in a recent study by Quirke $e t a l,{ }^{6}$ in which they reported that (1) full-length PPAD is autocitrullinated when artificially expressed in Escherichia coli, and (2) antibodies directed against glutathione S-transferase (GST)-tagged PPAD appeared to show preferential reactivity towards their autocitrullinated protein in $38 \%$ of patients with RA. While compelling as a model, their study did not address the nature of PPAD in $P$ gingivalis.

By studying PPAD directly within cultured $P$ gingivalis, we found that PPAD was not autocitrullinated in bacterial cells (nor as a secreted enzyme in cell supernatants). ${ }^{1}$ PPAD was expressed as a truncated protein when analysed in its native bacterial species, ${ }^{1}$ raising questions about the biological relevance of the 'full-length' PPAD proenzyme. To reconcile the absence of PPAD autocitrullination in $P$ gingivalis with the previous report of Quirke $e t a l$, we expressed the mature protein and the full-length proenzyme in E coli, and demonstrated that the truncated enzyme present in $P$ gingivalis cannot autocitrullinate. ${ }^{1}$ If autocitrullination is indeed absent in vivo, a citrulline-specific antibody response to PPAD in patients with RA cannot be expected (unless the result of anti-citrullinated protein antibody (ACPA) cross-reactivity). Testing this hypothesis, we demonstrated that antibodies in patients with RA cannot distinguish autocitrullinated and native PPAD by two independent techniques, ELISA and immunoprecipitation. ${ }^{1}$ Moreover, differential reactivity to PPAD by individual patient
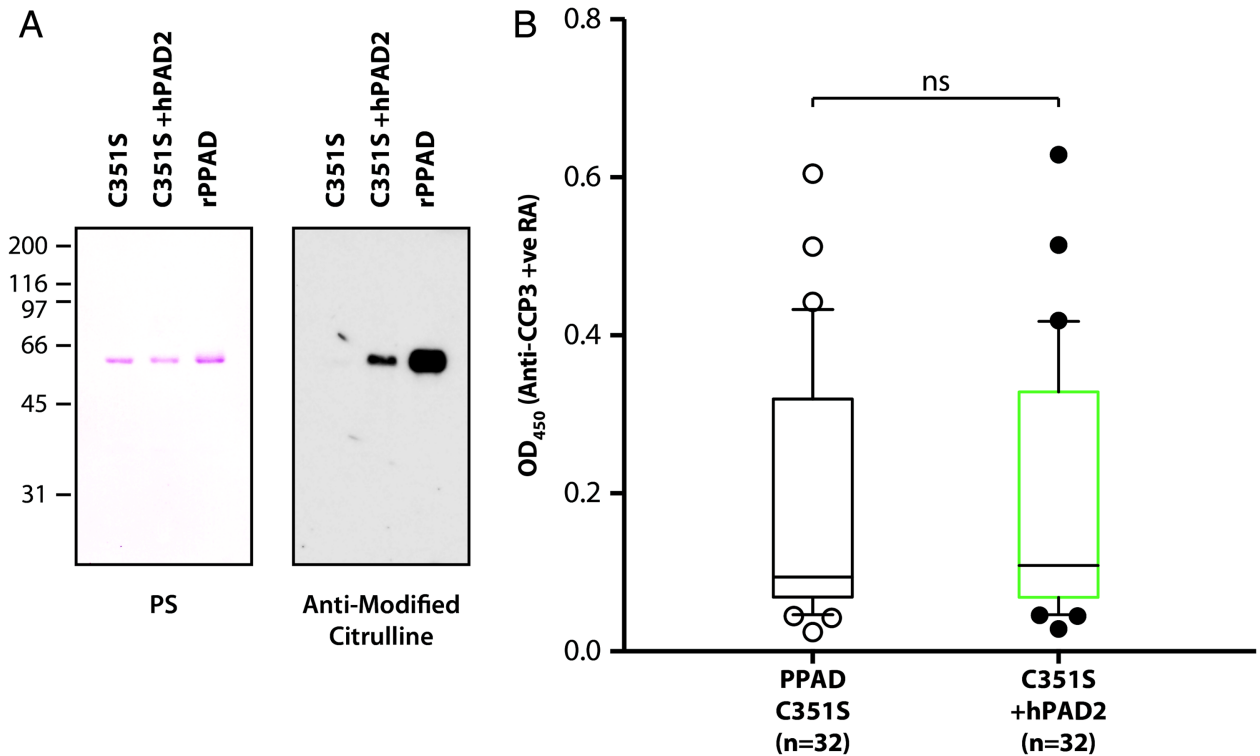

Figure 1 rPPAD is citrullinated by human PAD2 in vitro, but not preferentially recognised as a PAD2-citrullinated protein in anti-CCP positive rheumatoid arthritis (RA). (A) Native PPAD ${ }^{\text {C351S }}$ was incubated with 1000nM of human PAD2 in the presence of $10 \mathrm{mM}$ calcium chloride and 5 mM DTT. Native and in vitro citrullinated PPAD ${ }^{C 3515}$ were analysed by sodium dodecyl sulfate polyacrylamide gel electrophoresis (SDS-PAGE) and anti-modified citrulline (AMC) immunoblotting to detect citrullination (right panel). Ponceau staining (PS) before antibody probing is shown to visualise loading (left panel). Autocitrullinated rPPAD expressed in Escherichia coli served as a positive control. (B) Antibodies to unmodified PPAD $^{C 3515}$ and PPAD ${ }^{C 3515}$ citrullinated by human PAD2 in anti-CCP positive RA $(n=32)$. ELISA plates were coated with $100 \mathrm{ng}$ of unmodified or citrullinated PPAD ${ }^{\mathrm{C} 351 \mathrm{~S}}$, respectively, and optical densities (ODs) corrected for background reactivity of sera against human PAD alone. Horizontal lines indicate median reactivity for each group; whiskers depict the 10th and 90th centiles, respectively. Statistical analysis was performed using Wilcoxon matched-pairs signed rank test ( $n s=$ not significant; $p>0.05$ ). CCP, cyclic citrullinated peptide; DTT, dithiothreitol; PAD, peptidylarginine deiminase; $r P P A D$, recombinant Porphyromonas gingivalis peptidylarginine deiminase. 
sera was lost by SDS-PAGE immunoblotting, suggesting that the epitope recognised by anti-PPAD antibodies is conformationdependent (unpublished data).

As noted by Quirke et al, this absence markedly contrasts their findings of citrulline-specific anti-PPAD antibodies in RA. We hypothesised that this fundamental difference in the two PPAD ELISAs may relate to citrullination of the GST-protein tag linked to PPAD in the recombinant protein used by Quirke et $a l .{ }^{6}$ Unfortunately, this was not addressed in the authors' comment, ${ }^{2}$ nor did they provide evidence that PPAD autocitrullination occurs in their wild type strains of $P$ gingivalis. Instead, the authors revert to a synthetic citrullinated peptide (CPP3) to support an immune response to citrullinated PPAD in RA. ${ }^{2}$

We do not share Quirke et al's experience regarding the reproducibility of protein-based ELISAs, nor can we confirm that ELISAs using whole proteins in their native tertiary structure are an unreliable means to detect ACPAs. These assertions are somewhat surprising considering that the initial observations by Quirke $e t a l^{6}$ were based on full-length PPAD ELISAs, which performed favourably compared with their peptide assays $(38 \%$ vs $15 \%$ specific reactivity in RA). ${ }^{2}{ }^{6}$ The use of cyclic citrullinated peptides of PPAD may pose additional questions about the specificity of the observed antibody reactivity. Indeed, some cyclic citrullinated peptides are highly cross-reactive with ACPAs, which explains the success of the first cyclic citrullinated peptide derived from filaggrin (CCP1) to detect ACPAs by ELISA. ${ }^{7}$ In the absence of a gold standard to confirm specificity, PPAD CPP3 may only represent an additional surrogate antigen to capture ACPAs.

This is supported by our preliminary studies, in which we identified by immunoprecipitation that binding of anti-PPAD antibodies in patients with RA is restricted to a C-terminal region of PPAD (data not shown), mapping the epitope(s) recognised by anti-PPAD antibodies to a region of the enzyme distinct from the peptide sequences deemed immunodominant by Quirke et al (CPP3, CPP5 and CPP8). Thus, we suggest that the positive reactivity observed by the authors for 10 out of the 13 synthetic peptides tested should be interpreted with caution, and is by no means confirmatory. ${ }^{6}$

Lastly, a scenario in which PPAD is enzymatically modified by human PADs during periodontal infection is conceivable. However, it is important to emphasise that this does not represent autocitrullination. We addressed this hypothesis by citrullinating native PPAD ${ }^{\mathrm{C} 351 \mathrm{~S}}$ (a mutant protein that is not artificially autocitrullinated in vitro) using human recombinant PAD2. Citrullination of PPAD ${ }^{\mathrm{C} 351 \mathrm{~S}}$ by human PAD2 was confirmed by anti-modified citrulline immunoblotting (figure 1A), and not observed with human PAD3 and PAD4 (data not shown). Using native and citrullinated PPAD ${ }^{\mathrm{C} 351 \mathrm{~S}}$, we analysed sera from 32 patients with RA who were anti-cyclic citrullinated peptide antibody positive by ELISA. Anti-PPAD antibodies did not show heightened reactivity against PAD2-citrullinated $\mathrm{PPAD}^{\mathrm{C} 351 \mathrm{~S}}$ as compared with unmodified PPAD ${ }^{\mathrm{C} 351 \mathrm{~S}}$ (mean optical density 0.164 vs $0.161 ; p=0.22$ ), suggesting that anti-PPAD antibodies bind PPAD independent of citrullination (figure 1B). This is in accordance with our previous data using in vitro autocitrullinated $\mathrm{rPPAD},{ }^{1}$ reinforcing the absence of citrulline-specific anti-PPAD responses in RA. Importantly, ACPAs were detected in this assay using native versus citrullinated vimentin as positive control (data not shown). In the absence of a citrulline-specific anti-PPAD response, citrullination of PPAD by human PADs in vivo remains speculative and cannot suggest a mechanistic role for PPAD as a citrullinated antigen relevant to the initiation of autoimmunity in RA.
Elucidating the role of periodontal infection as a potential trigger of autoimmunity in RA is of great interest. We agree that to establish PPAD autocitrullination in vivo, mass spectrometric data of $P$ gingivalis during human infection is indispensable. In the meantime, we suggest that studying PPAD in $P$ gingivalis cells is a better approximation than the recombinant expression of a putative isoform in E coli. PPAD remains a candidate for inducing autoimmunity in RA, likely by citrullinating antigens, but not through autocitrullination. While PPAD has provided a beautiful model to explain loss of tolerance in RA, we suggest that PPAD autocitrullination, and the citrullination of PPAD by human PADs addressed in this study, are not aetiologically linked to RA.

\section{Maximilian F Konig, Clifton 0 Bingham III, Felipe Andrade}

Division of Rheumatology, The Johns Hopkins University School of Medicine, Baltimore, Maryland, USA

Correspondence to Dr Felipe Andrade, Division of Rheumatology, Johns Hopkins University School of Medicine, Mason F. Lord Building Center Tower, Suite 6000 5200 Eastern Avenue, Baltimore, MD 21224, USA; andrade@jhmi.edu

Acknowledgements The authors thank Janelle M Montagne (Division of Rheumatology, The Johns Hopkins University School of Medicine) for critical review of the manuscript.

Contributors Each author has contributed to one or more of the following aspects of the manuscript-conception and design, acquisition, analysis and interpretation of data, drafting and revising the article.

Funding The Donald B and Dorothy L Stabler Foundation, The Mackley Fund from Sibley Memorial Hospital, The Johns Hopkins Arthritis Center Research Fund, and The Jerome L Greene Foundation Scholar Award.

\section{Competing interests None.}

Ethics approval Johns Hopkins Medicine Institutional Review Board.

Provenance and peer review Commissioned; internally peer reviewed.

Data sharing statement All data relevant for this study have been included in the manuscript. Any additional data referred to as not shown will be available through direct contact with the corresponding author. Reagents derived from this project will be available upon request through material transfer agreements.

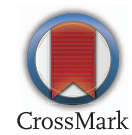

To cite Konig MF, Bingham CO 3rd, Andrade F. Ann Rheum Dis 2015;74:e8.

Received 12 October 2014

Accepted 20 October 2014

Published Online First 7 November 2014

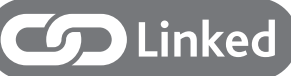

http://dx.doi.org/10.1136/annrheumdis-2014-206665

Ann Rheum Dis 2015;74:e8. doi:10.1136/annrheumdis-2014-206681

\section{REFERENCES}

1 Konig MF, Paracha AS, Moni M, et al. Defining the role of Porphyromonas gingivalis peptidylarginine deiminase (PPAD) in rheumatoid arthritis through the study of PPAD biology. Ann Rheum Dis 2014. Published Online First: 26 May 2014. doi:10.1136/ annrheumdis-2014-205385

2 Quirke A-M, Lundberg K, Potempa J, et al. PPAD remains a credible candidate for inducing autoimmunity in rheumatoid arthritis: comment on the article by Konig et al. Ann Rheum Dis 2015;74:e7.

3 Schellekens GA, de Jong BA, van den Hoogen FH, et al. Citrulline is an essential constituent of antigenic determinants recognized by rheumatoid arthritis-specific autoantibodies. J Clin Invest 1998;101:273-81.

4 Rosenstein ED, Greenwald RA, Kushner L, et al. Hypothesis: the humoral immune response to oral bacteria provides a stimulus for the development of rheumatoid arthritis. Inflammation 2004;28:311-18. 
5 Wegner N, Wait R, Sroka A, et al. Peptidylarginine deiminase from Porphyromonas gingivalis citrullinates human fibrinogen and $\alpha$-enolase: Implications for autoimmunity in rheumatoid arthritis. Arthritis Rheum 2010;62:2662-72.

6 Quirke A-M, Lugli EB, Wegner N, et al. Heightened immune response to autocitrullinated Porphyromonas gingivalis peptidylarginine deiminase: a potential mechanism for breaching immunologic tolerance in rheumatoid arthritis. Ann Rheum Dis 2014;73:263-9.

7 Schellekens GA, Visser $\mathrm{H}$, de Jong BA, et al. The diagnostic properties of rheumatoid arthritis antibodies recognizing a cyclic citrullinated peptide. Arthritis Rheum 2000;43:155-63. 